Check for updates

Cite this: Mater. Adv., 2021,

2, 5057

Received 31st May 2021,

Accepted 6th July 2021

DOI: $10.1039 / \mathrm{d} 1 \mathrm{ma} 00480 \mathrm{~h}$

rsc.li/materials-advances

\section{Photoactuation of micromechanical devices by photochromic molecules $\dagger$}

\author{
José Elías Angulo-Cervera, (D) ab Mario Piedrahita-Bello, ${ }^{\text {ab }}$ Barbora Brachňaková, ${ }^{a c}$ \\ Alejandro Enríquez-Cabrera, (D) a Liviu Nicu, ${ }^{b}$ Thierry Leichle, ${ }^{\text {bd }}$ Fabrice Mathieu, ${ }^{\text {b }}$ \\ Lucie Routaboul, ${ }^{a}$ Lionel Salmon, (D) a Gábor Molnár (iD *a and \\ Azzedine Bousseksou*a
}

\begin{abstract}
Spiropyran doped P(VDF-TrFE) nanocomposite films were spraycoated onto silicon micro-cantilevers. We show that switching the molecules from the closed- to the open-ring form by UV light gives rise to a downward bending of the cantilever as well as to a decrease of its resonance frequency. This photoactuation is reversible by means of heating and simultaneous visible light irradiation. From the flexural bending and resonance data, we extracted the actuation strain $(0.13 \pm 0.03 \%)$, stress ( $4 \pm 1 \mathrm{MPa}$ ) and elastic energy density $\left(2.3 \pm 1 \mathrm{~mJ} \mathrm{~cm}^{-3}\right)$ associated with the photoisomerization of spiropyran molecules in the composite films.
\end{abstract}

Photoactuation (or photostriction) refers to various physical phenomena, which allow converting light energy into mechanical work and motion. Light is an appealing stimulus for mechanical actuating purposes because it allows for the remote control of the actuator (without wiring) with high temporal and spatial resolutions. In addition, the unique properties of the light wave (wavelength selectivity, polarization state, coherence, etc.) may be beneficial to achieve complex functionalities. Last, but not least there is also an interest due to the possibility of renewable solar energy use.

From a broad perspective, the photoactive agent in photoactuators can be inorganic or organic, which can trigger diverse phenomena such as desorption, phase transition, photo-thermal expansion and photochemical reaction. ${ }^{1-6}$ Among these photostrictive materials, photochromic molecules (azobenzenes, stilbenes, diarylethenes,

\footnotetext{
${ }^{a}$ LCC, CNRS \& University of Toulouse, 205 route de Narbonne, Toulouse 31077, France.E-mail: azzedine.bousseksou@lcc-toulouse.fr gabor.molnar@lcc-toulouse.fr

${ }^{b}$ LAAS, CNRS \& University of Toulouse, 7 avenue du Colonel Roche, Toulouse 31400, France

${ }^{c}$ Department of Inorganic Chemistry, Faculty of Chemical and Food Technology, Slovak University of Technology in Bratislava, Bratislava SK-81237, Slovakia

${ }^{d}$ Georgia Tech-CNRS International Research Laboratory,

School of Electrical and Computer Engineering, Atlantic Drive, Atlanta, GA, 30332, USA

$\dagger$ Electronic supplementary information (ESI) available: Additional experimental details and sample characterization data. See DOI: 10.1039/d1ma00480h
}

fulgides, etc.) have received much attention since the first reports in the 70 s. $^{7-12}$ These photochromic molecules exhibit in many cases wavelength-selective, two-way ON/OFF photoswitching between a stable and a metastable molecular configuration, which may occur due to photoisomerization (e.g. cis-trans isomerization, keto-enol tautomerism, ring opening/closure) or photodimerisation phenomena. ${ }^{13,14}$ Crucially, these systems can be maintained in the metastable state for some time (up to the 1000 years range) without consuming any energy. ${ }^{15,16}$

It is important to note, however, that photoswitching in bulk photochromic materials is often difficult to achieve due to the close packing of molecules, which impedes structural changes. In addition, light penetration is often very incomplete in bulk materials, restricting the excitation to the uppermost layers. Finally, yet crucially, the integration of bulk samples into functional devices is not straightforward. These problems have been effectively alleviated by incorporating the photochromic species into different soft matrices, including polymers, liquid crystal elastomers and gels, leading to countless examples of ingenious, macroscopic photo-actuators. ${ }^{1-5,17-20}$ On the other hand, much less efforts have been devoted to integrate photochromic molecules into micro/nano-scale mechanical devices. To our best knowledge, MEMS-type microactuators have been functionalized by photochromic compounds only by two groups. Using an adhesive layer, Jackson et al. ${ }^{21}$ bonded freestanding photo-responsive liquid-crystal polymer films onto silicon and Parylene-C cantilevers. They demonstrated a lightinduced, reversible downshift of the MEMS resonance frequency (ca. 10\%), which they attributed to the lower elastic modulus of the photoisomerized polymer film. On the other hand, Grogan et al. ${ }^{22,23}$ have functionalized gold-coated silicon microcantilevers with a spiropyran monolayer by immersing the devices into the solution of the molecules. When irradiated by UV and visible light sources the microcantilever showed a reversible deflection, which was assigned to surface stress changes induced by the photoisomerization.

The key enabling issue for high-performance microactuators is the quality of the photochromic film in terms of uniformity 
and mechanical properties. Unfortunately, conventional solutionprocessing methods, such as spin coating and solvent casting, produce good results primarily on smooth surfaces. As such, these liquid-phase methods are not well adapted for the integration of thin films into MEMS devices, which exhibit a complex 3D geometry with deep topography features, such as trenches, grooves and cavities. In particular, drying the liquid often results in the MEMS components "sticking" to the substrate rather than being suspended. In this context, the so-called 'spray coating' technique, which consists in spraying a solution by a pressurized gas onto a substrate through a nozzle, is known to be a viable approach for film deposition on MEMS devices. ${ }^{24-26}$ Here, we use this approach to coat silicon microcantilevers by micro- and nanometric films of photochromic molecules embedded in a polymer matrix. We show that the photochromic composite can be used to tune the mechanical properties of the device as well as to perform controlled and reversible photo-induced motion.

For this study we use the commercial $1^{\prime}, 3^{\prime}$-dihydro- $1^{\prime}, 3^{\prime}, 3^{\prime}$ trimethyl-6-nitrospiro( $2 H$-1-benzopyran-2,2'- $(2 H)$-indole) molecule as active material and the copolymer poly(vinylidene fluoride-trifluoro-ethylene), P(VDF-TrFE), with 70-30\% monomer ratio, as a polymer matrix. We have chosen this well-known photochromic molecule because it displays a UV-light-induced switching from the closed-ring spiropyran (SP) form to an openring merocyanine (MC) isomer, which can be reversed by heating and/or visible light irradiation (see Scheme 1 and Fig. S1 in the ESI $\dagger$ ). Importantly for us, the SP to MC isomerization is associated with a sizeable strain, the planar MC isomer occupying $c a$. $1 \%$ more volume (Fig. S2, ESI $\dagger$ ). An acetone/2-butanone solution of $\mathbf{S P}$ and $\mathrm{P}(\mathrm{VDF}-\mathrm{TrFE})$ was spray-coated over silicon and glass substrates (held at $65{ }^{\circ} \mathrm{C}$ ) using a SUSS MicroTec AltaSpray instrument. Films with nominally $33 \mathrm{wt} \%$ filler content were obtained by this method with various thicknesses from $\mathrm{ca} .300 \mathrm{~nm}$ to $30 \mu \mathrm{m}$, depending on the number of coating cycles and the flow rate. While seemingly straightforward, spraying of solutions on surfaces is not necessarily simple. High flow rates led to a solvent "flooding" over the substrate with subsequent non-homogeneous films (Fig. 1a), whereas low rates gave rise to early solvent evaporation that generates spider-web-like fibres (Fig. 1b). Critically, when deposited directly onto glass or silicon substrates, numerous micrometre-sized, faceted SP crystals formed together with the composite film (Fig. 1c and see also Fig. S3, ESI $\dagger$ ). Since this phenomenon occurred predominantly during the first coating cycle (presumably due to the different surface wettabilities), we realized that it can be readily suppressed by pre-coating the

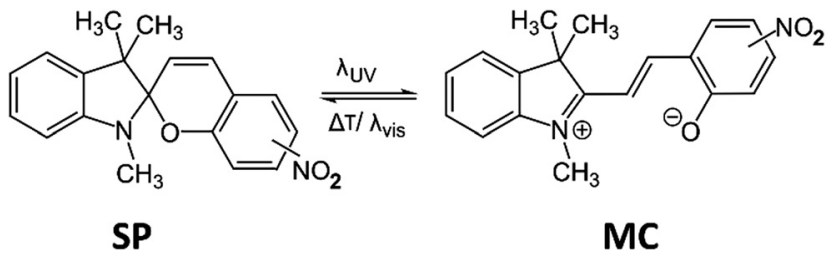

Scheme 1 Photoisomerization of the spiropyran molecule used in this work.

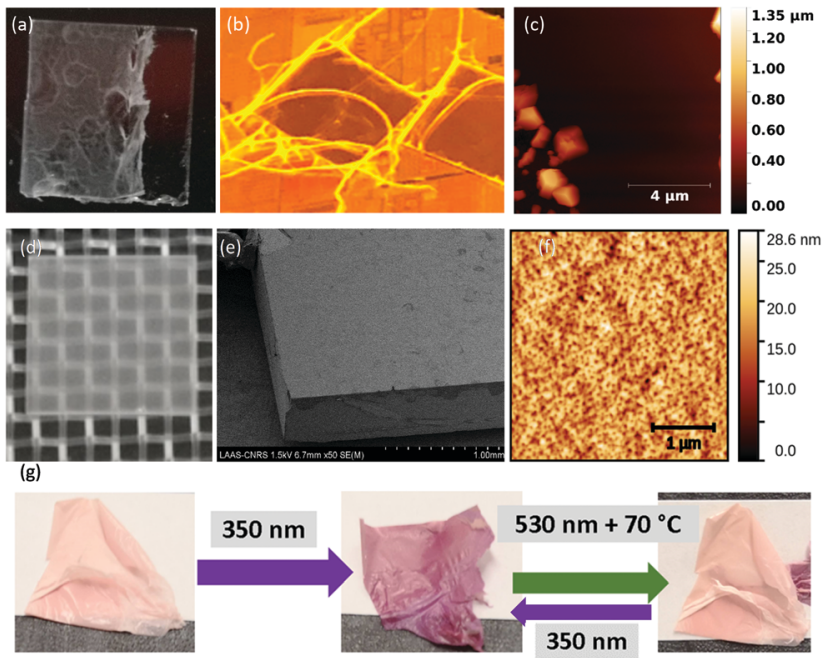

Fig. 1 Spray coating of spiropyran(P(VDF-TrFE) composite films. Effects of (a) low or (b) high flow rates and (c) lack of surface treatment on the film morphology. (d) Optical microscopy, (e) SEM and (f) AFM topography images of a $300 \mathrm{~nm}$ thick composite film deposited on a P(VDF-TrFE) pre-coated glass substrate with optimized coating parameters. (g) Photographs of a $30 \mu \mathrm{m}$ thick, freestanding film before and after UV irradiation as well as after successive visible light irradiation at $70{ }^{\circ} \mathrm{C}$.

substrates with a $200 \mathrm{~nm}$ thin $\mathrm{P}(\mathrm{VDF}-\mathrm{TrFE})$ layer. The composite films subsequently deposited on top of these pre-coated substrates do not contain such crystals (Fig. 1d). Indeed, scanning electron microscopy (SEM) and atomic force microscopy (AFM) images of these optimized films reveal a smooth surface for each film thickness (Fig. 1e and f). Infrared spectroscopic fingerprints of the composite films and the pure SP powder confirm the incorporation of SP molecules within the composite (Fig. S4, ESI $\dagger$ ). Grazing-incidence X-ray diffraction (GIXRD) revealed that the incorporated photochromic material is nanocrystalline and the crystallites display a preferential orientation with their (202) planes parallel to the substrate surface (Fig. S5, ESI $\dagger$ ). The nanocrystals cannot be discerned in SEM or AFM images (Fig. S6, ESI $\dagger$ ), but their size could be estimated as $\sim 30 \mathrm{~nm}$ from the GIXRD data using the Scherrer equation. Interestingly, films above a few microns thickness, deposited on antiadhesive substrates (e.g. Teflon), can be peeled off (Fig. 1g).

Photo-isomerization of the composite films was triggered by irradiating them with LED light sources emitting at $350 \mathrm{~nm}$ $\left(1.1 \mathrm{~mW} \mathrm{~mm}^{-2}\right)$ and $530 \mathrm{~nm}\left(0.6 \mathrm{~mW} \mathrm{~mm}^{-2}\right)$ wavelengths. The thickest films $(>10 \mu \mathrm{m})$ were tested only for their reversible perceptible colour change (Fig. 1g). The initially pale orange composites develop a deep purple colour after being irradiated by UV light, which can be reversed upon visible light irradiation at high temperature. The photochromic properties of the thinner films were assessed quantitatively by a UV-Vis spectrophotometer. Fig. 2 displays the data acquired for a $1500 \mathrm{~nm}$ thick film, whereas comparable results for other thicknesses (375 and $750 \mathrm{~nm}$ ) are shown in Fig. S7 (ESI $\dagger$ ). A first spectrum was acquired in the dark, just after mounting the sample on the heating-cooling stage. As expected for the closed-ring isomer, 

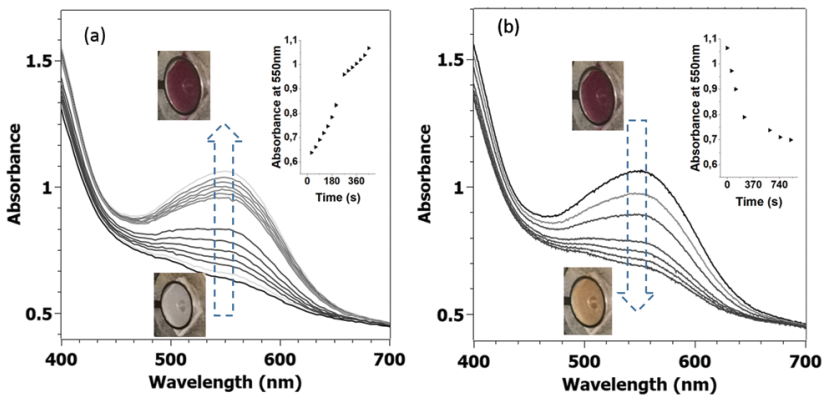

Fig. 2 Photochromic properties of the composite films. UV-VIS absorption spectra of a $1550 \mathrm{~nm}$ thick spiropyran (PP(VDF-TrFE) film. (a) Successive stages of the isomerization of the as-synthesized film into the open-ring form under UV irradiation at $25^{\circ} \mathrm{C}$. (b) Back conversion to the closed-ring form by green light irradiation at $70{ }^{\circ} \mathrm{C}$. The inserts show the temporal evolution of the absorbance at $550 \mathrm{~nm}$ for the forward and reverse conversions. The photographs show the sample before and after the UV and visible light irradiations.

this spectrum showed no absorption in the visible spectral range. The film was then irradiated at $350 \mathrm{~nm}$ and a spectrum was acquired every 30 seconds. Fig. 2a displays these spectra, where the emergence of an absorption band centred at $550 \mathrm{~nm}$ is clearly observed. For the 375 and $750 \mathrm{~nm}$ thick samples, the absorbance reached saturation following $c a$. 1.5 and $4 \mathrm{~min}$ respectively (see Fig. S7 and also S8, ESI $\dagger$ for a comparison with solution kinetics). For the thicker, $1500 \mathrm{~nm}$ film, saturation could not be reached even after $8 \mathrm{~min}$ exposure. Following the UV irradiation, the film was irradiated at $530 \mathrm{~nm}$, but the absorption spectrum showed no variation for several hours of constant irradiation. We noticed also that the coloured films showed no fading for several days in ambient conditions. In other words, the open-ring isomer, which has a lifetime of only a few minutes in solution, has been stabilized in the composite to such extent that return to the ground state cannot be achieved at room temperature. We hypothesize that this stabilization of the $\mathbf{M C}$ form in the nanocomposite films might arise due to electrostatic interactions with the polar $\mathrm{P}(\mathrm{VDF}-\mathrm{TrFE})$ matrix. We realized, however, that the reverse process (MC to SP) could be accomplished at $70{ }^{\circ} \mathrm{C}$ under $530 \mathrm{~nm}$ irradiation. Yet, as shown in Fig. 2b, this is a slower and less efficient process when compared to the SP to $\mathbf{M C}$ isomerization and even after $25 \mathrm{~min}$ irradiation there remains $\mathrm{ca}$. 20\% MC isomer in the film. This residual fraction is most likely related to the intrinsic heterogeneity of the composite made of a semicrystalline matrix. It is also worth to note that both the forward and the (incomplete) back conversions are perceptible by the naked eye (see photographs in Fig. 2).

Aiming for the integration of the photochromic composites into MEMS devices, we fabricated silicon microcantilevers (150 $\mu \mathrm{m}$ length, $50 \mu \mathrm{m}$ width and $2 \mu \mathrm{m}$ thickness), which were spray-coated with the composite films $(375,750$ and $1500 \mathrm{~nm}$ thickness) in the last fabrication step (Fig. 3a and b). Details of the MEMS fabrication and operation are given in ref. 27-29. The dynamic and static behaviours of the cantilevers were tracked simultaneously to assess in real time the resonance frequency $\left(f_{\mathrm{r}}\right)$ and the quality factor $(Q)$ of the cantilever, as well
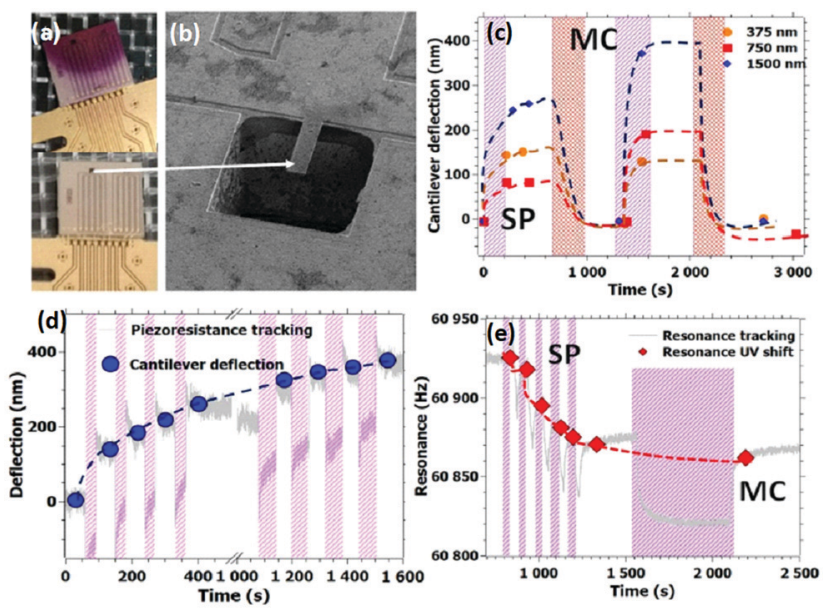

Fig. 3 Photoactuation of MEMS devices. (a) Photographs of the MEMS device coated with a $1500 \mathrm{~nm}$ thick spiropyran (PP(VDF-TrFE) film before (colorless) and after (violet) UV irradiation. (b) SEM image of the MEMS cantilever. (c) MEMS cantilever deflection during two successive irradiation cycles under UV light at $25^{\circ} \mathrm{C}$ (violet shaded areas) and under subsequent visible light irradiation at $70{ }^{\circ} \mathrm{C}$ (red shaded areas) recorded for three different film thicknesses (375, 750 and $1500 \mathrm{~nm}$ ). The dashed lines are guides to the eye. (d) MEMS cantilever deflection and (e) MEMS resonance frequency tracking for successive ON/OFF UV irradiation cycles (violet shaded areas) at $25^{\circ} \mathrm{C}$ for a $1500 \mathrm{~nm}$ thick film. The dashed lines are guides to the eye to follow the persistent photo-effects.

as the amplitude of the cantilever tip deflection $(\delta)$. The measurements were conducted at a controlled temperature and constant vacuum pressure of $10 \mathrm{mbar}$, which allows for high quality factors and shunts parasitic signals due to changes in the environmental conditions. The cantilevers used in this work were fabricated from p-type, doped silicon. This type of semiconductor material is known to be responsive to light irradiation. ${ }^{30}$ In order to discriminate the photo-induced signals arising from the silicon cantilever and from the composite films, we have assessed the properties of the uncoated cantilevers. Crucially, the photo-induced response of the uncoated cantilever was found reversible both in the UV and visible spectral ranges (Fig. S9 and S10, ESI $\dagger$ ), apart from a small drift of $f_{\mathrm{r}}$ during the first UV exposure, allowing thus to distinguish in an unequivocal manner the effects of the photochromic coating, which persist even after the light irradiation is turned off.

To evaluate the photoactuation properties of our composite films, a series of ON-OFF irradiation cycles were carried out using UV $\left(\lambda=350 \mathrm{~nm}, T=25^{\circ} \mathrm{C}\right)$ and visible light $(\lambda=530 \mathrm{~nm}$, $\left.T=70{ }^{\circ} \mathrm{C}\right)$ irradiation sequences. The tracking of static and dynamic properties of the MEMS is shown in Fig. 3c-e (see also Fig. S11 and S12, ESI $\dagger$ ). In the static regime, we can notice the cantilever bending produced by the UV photo-isomerization in the composite layer, which was found additive upon successive short irradiation cycles (Fig. 3d) and reversible under visible light irradiation at $70{ }^{\circ} \mathrm{C}$ (Fig. 3c). We can thus unambiguously ascribe this static bending to the UV photo-isomerization of SP to $\mathbf{M C}$ in the composite films. Since upon UV irradiation we detected downward bending of the cantilever, we can conclude 
that the SP to MC switch produces a volume expansion of the composite layer (i.e. a tensile strain), which leads to photoactuation. This finding is perfectly in line with the results of Grogan et al. ${ }^{22,23}$ reported for silicon cantilevers grafted by a spiropyran monolayer. These authors have observed also a downward (upward) cantilever deflection upon the SP to MC (MC to SP) isomerization. Contrary to their experiments, however, in our case subsequent visible light irradiation cycles allowed to recover the initial closed-ring form only at $70{ }^{\circ} \mathrm{C}$. This difference between the two experiments is most likely related to the different chemical composition and/or spatial arrangement of the SP molecules.

In the dynamic regime, UV irradiation produced a decrease in the resonance frequency (Fig. 3e) with concomitant small changes in the quality factor (Fig. S11, ESI $\dagger$ ). Whereas the changes of the $Q$ factor are not lasting, the effect of the UV light on the resonance frequency is persistent (ca. $60 \mathrm{~Hz}$ shift between the SP and MC forms for the 750 and $1500 \mathrm{~nm}$ thick films) and no spontaneous reversion is observed. Similar to the effect of UV light on the static deflection, the resonance frequency changes are cumulative for subsequent irradiation cycles (until saturation) and can be erased by visible light irradiation at $70{ }^{\circ} \mathrm{C}$. The decrease of the resonance frequency when going from the closed to the open-ring form could be attributed to the concomitant decrease of the elastic modulus, although changes of the film thickness and density might also influence the dynamic behaviour.

The combined cantilever deflection and resonance data were used to extract the photoactuation parameters of the composite films (see Table S1, ESI $\dagger$ ). Using the flexural resonance approach described in ref. 31, the resonance frequency difference between the uncoated and coated cantilevers ( $c a .10 \mathrm{kHz}$ ) provides an estimated Young's modulus of $E=3.0 \mathrm{GPa}$ for the composite film. This value of $E$ together with the cantilever geometry and static deflection data were used as inputs for Thimoshenko's equation, ${ }^{32}$ which allowed us to calculate the photoactuation strain $(\varepsilon=0.10-0.16 \%$, depending on the film thickness). The actuation stress was estimated using the Stoney formula $^{33}(\sigma=3.3-5.1 \mathrm{MPa}$, depending on the film thickness). Finally, the volumetric elastic energy density (work, $W$, per volume unit, $V$ ) of the composite film was calculated as $W / V=E \varepsilon^{2} / 2$, with $E$ the Young's modulus and $\varepsilon$ the strain of the film. The obtained work density values $\left(1.6-3.6 \mathrm{~mJ} \mathrm{~cm}^{-3}\right)$ are comparable in magnitude with previously reported polymer composite photochromic actuators. ${ }^{20,34}$

In summary, we spray-coated photochromic nanocomposite films, based on a P(VDF-TrFE) matrix with 33 wt\% spiropyran load. We obtained high quality films, which are smooth and homogeneous and whose thickness can be adjusted in a broad range $(c a .0 .3-30 \mu \mathrm{m})$. The films preserve the photochromic properties of spiropyran regardless of their thickness: they can be switched between a pale orange and a deep purple color, associated with the photo-isomerization between the closedand open-ring forms of the photoactive molecules. Whereas switching to the open-ring isomer was straightforward using UV light irradiation, the reverse process could be achieved by visible light irradiation only at high temperatures $\left(70{ }^{\circ} \mathrm{C}\right)$. The films were deposited over silicon microcantilevers to produce a bilayer architecture. We have shown that the switching of the active molecules in the composite films from the closed to the open-ring form by UV light gives rise to a downward bending of the cantilever as well as to a decrease of its resonance frequency. The combined analysis of flexural bending and resonance data allowed us to assess the relevant photoactuation parameters of the films. The approach we used here is highly versatile and can afford for the integration of micromechanical systems by various photochromic molecules and polymer matrices. The high quality of the films produced by spray-coating enables the quantitative analysis of their mechanical/actuation properties and open up an appealing scope for their application for photoactuation and in situ resonance tuning/calibration purposes. Further work should focus on the optimization of the chemical composition, concentration and size of the photochromic particles in order to increase the performance metrics (actuation amplitude, speed, stability, etc.).

This work was supported by the Occitanie Region/Federal University of Toulouse ( $\mathrm{PhD}$ grant of MPB), the CONACyTMexico (PhD grant 471690 of JEAC), the National Scholarship Programme of the Slovak Republic for the Support of Mobility of Students, PhD Students, University Teachers, Researchers and Artists 2020; APVV-19-0087; VEGA 1/0125/18; APVV-18-0197 (grants of BB), the Agence Nationale de la Recherche (ANR-19CE09-0008-01) and by the LAAS-CNRS technology platform, a member of Renatech network.

\section{Author's contributions}

JEAC: film preparation and characterization (AFM, SEM, UV-VIS, MEMS), paper writing, MPB: spray coating experiments, BB: solution and solid state experiments (Uv-VIS, XRD), AEC: molecular simulations, LN: conceptualization, funding acquisition, TL: supervision of clean room work, MF: supervision of MEMS characterisation, LR: supervision of sample preparation, LS: supervision of sample characterization, GM: conceptualisation, paper writing, AB: project management, funding acquisition. All authors has discussed the experimental plans, contributed to data analysis and participated to the editing of the final draft of the manuscript.

\section{Conflicts of interest}

There is no conflicts of interest to declare.

\section{Notes and references}

1 Y. Chen, et al., Mater. Horiz., 2021, 8, 728.

2 J. Li, X. Zhou and Z. Liu, Adv. Opt. Mater., 2020, 8, 2000886.

3 M. Kondo, Polym. J., 2020, 52, 1027.

4 Y. Hu, Z. Li, T. Lan and W. Chen, Adv. Mater., 2016, 28, 10548. 
5 P. Naumov, S. Chizhik, M. K. Panda, N. K. Nath and E. Boldyreva, Chem. Rev., 2015, 115, 12440.

6 T. J. White, Photomechanical Materials, Composites, and Systems: Wireless Transduction of Light into Work, John Wiley \& Sons Ltd, Chichester, UK, 2017.

7 G. Van Der Veen and W. Prins, Nat. Phys. Sci., 1971, 230, 70.

8 F. Agolini and F. P. Gay, Macromolecules, 1970, 3, 349.

9 G. Smets and F. de Blauwe, Pure Appl. Chem., 1974, 39, 225.

10 C. D. Eisenbach, Polymer, 1980, 21, 1175.

11 M. Irie and D. Kungwatchakun, Makromol. Chem., Rapid Commun., 1985, 5, 829.

12 H. Finkelmann, E. Nishikawa, G. G. Pereira and M. Warner, Phys. Rev. Lett., 2001, 87, 015501.

13 B. L. Feringa and W. R. Browne, Molecular Switches, Wiley-VCH Verlag \& Co. KGaA, Weinheim, Germany, 2011.

14 H. Tian and J. Zhang, Photochromic Materials: Preparation, Properties and Applications, Wiley-VCH Verlag GmbH \& Co. KGaA, Weinheim, Germany, 2016.

15 H. Qian, S. Pramanik and I. Aprahamian, J. Am. Chem. Soc., 2017, 139, 9140.

16 P. Naumov, P. Yu and K. Sakurai, J. Phys. Chem. A, 2008, $112,5810$.

17 T. Kim, L. Zhu, R. O. Al-Kaysi and C. J. Bardeen, ChemPhysChem, 2014, 15, 400.

18 A. Priimagi, C. J. Barrett and A. Shishido, J. Mater. Chem. C, 2014, 2, 7155.

19 T. Ikeda, J. Mamiya and Y. Yu, Angew. Chem., Int. Ed., 2007, 46, 506.
20 M. D. Manrique-Juárez, et al., Coord. Chem. Rev., 2016, 308, 395.

21 N. Jackson, K. Kumar, O. Olszewski, A. P. H. J. Schenning and M. G. Debije, Smart Mater. Struct., 2019, 28, 085024.

22 C. Grogan, et al., Sens. Actuators, B, 2016, 237, 479.

23 C. Grogan, et al., Sensors, 2020, 20, 854.

24 T. Luxbacher and A. Mirza, Sensors, 1999, 16, 61.

25 N. P. Pham, T. L. M. Scholtes, R. Klerk, B. Wieder, P. M. Sarro and J. N. Burghartz, Micromachining and Microfabrication Process Technology VII, 2001, vol. 4557, pp. 312-319.

26 L. Yu, Y. Y. Lee, F. E. H. Tay and C. Iliescu, J. Phys.: Conf. Ser., 2006, 34, 937.

27 D. Saya, T. Leïchlé, J. B. Pourciel, C. Bergaud and L. Nicu, J. Micromech. Microeng., 2007, 17, N1.

28 I. Dufour, et al., J. Sens., 2012, 719898.

29 M. D. Manrique-Juárez, et al., Adv. Funct. Mater., 2018, 28, 1801970.

30 Y. L. Guo, J. Zhou, Y. Huang and M. H. Bao, Sensors, 2007, 7, 1713.

31 M. D. Manrique-Juarez, et al., Appl. Phys. Lett., 2016, 109, 061903.

32 S. Timoshenko, J. Opt. Soc. Am., 1925, 11, 233.

33 G. G. Stoney, Proc. R. Soc. London, Ser. A, 1909, 82, 172.

34 J. Mahmoud Halabi, E. Ahmed, S. Sofela and P. Naumov, Proc. Natl. Acad. Sci. U. S. A., 2021, 118, e2020604118. 